\title{
Transmedullary Venous Anastomoses: Anatomy and Angiographic Visualization Using Flat Panel Catheter Angiotomography
}

L. Gregg and P. Gailloud

\begin{abstract}
SUMMARY: Flat panel catheter angiotomography, a recently developed angiographic technique, offers a spinal equivalent to the venous phase obtained during cerebral angiography. This report of 8 clinical cases discusses the flat panel catheter angiotomography appearance of a type of spinal venous structure until now principally known through the analysis of postmortem material, transmedullary venous anastomosis. The illustrated configurations include centrodorsolateral, median anteroposterior, median anteroposterior with duplicated origin, and combined centrodorsolateral/median anteroposterior transmedullary venous anastomoses, while a pathologic example documents the potential role of transmedullary venous anastomoses as collateral venous pathways. Two of the reported configurations have not been previously documented. Transmedullary venous anastomoses are normal venous structures that need to be differentiated from spinal cord anomalies, such as intramedullary vascular malformations.
\end{abstract}

ABBREVIATIONS: AMSV = anteromedian spinal vein; FPCA = flat panel catheter angiotomography; PLSV = posterolateral spinal vein; PMSV $=$ posteromedian spinal vein; TMVA = transmedullary venous anastomosis

$\mathbf{T}$ he clinical evaluation of the spinal venous system is technically challenging, even with spinal DSA, the criterion standard imaging technique for spinal vasculature. This suboptimal visualization correlates with a poor understanding of spinal venous system pathology. Flat panel catheter angiotomography (FPCA), a novel angiographic technique that offers a spinal equivalent to the cerebral venous phase, ${ }^{1-3}$ has shown great promise for the evaluation of the normal and abnormal spinal venous system..$^{3-5}$

This retrospective review of 8 clinical cases describes the morphology and angiographic appearance of several types of transmedullary venous anastomoses (TMVAs), including some previously undocumented configurations observed during routine spinal DSA. TMVAs, rarely observed in living subjects, ${ }^{6-8}$ were until now essentially known through the study of postmortem material.

\section{CASE SERIES}

\section{Acquisition Protocols}

Thoracic and lumbar protocols involved 20-second nonsubtracted rotational angiograms (Artis zee; Siemens, Erlangen, Germany) obtained during the selective injection of arteries provid-

Received November 19, 2014; accepted after revision January 13, 2015.

From the Division of Interventional Neuroradiology (L.G., P.G.), The Johns Hopkins Hospital, Baltimore, Maryland; and Department of Art as Applied to Medicine (L.G.), The Johns Hopkins University School of Medicine, Baltimore, Maryland.

Please address correspondence to Philippe Gailloud, MD, Division of Interventional Neuroradiology, The Johns Hopkins Hospital, Bloomberg 7218, 1800 Orleans St, Baltimore, MD 21287; e-mail: phg@jhmi.edu

http://dx.doi.org/10.3174/ajnr.A4302 ing major radiculomedullary branches. The contrast mixture (25\% contrast agent, $75 \%$ heparinized saline) was injected at a rate of $1 \mathrm{~mL} / \mathrm{s}$ for 30 seconds, covering the 20 -second angiography and a 10-second preacquisition delay (total volume of $30 \mathrm{~mL}$, including $7.5 \mathrm{~mL}$ of iodinated agent). The patients were not required to breath-hold during acquisitions. The volume and rate of injection were modified for vertebral artery and costocervical trunk studies $(50 \mathrm{~mL}$ at $2 \mathrm{~mL} / \mathrm{s}$, including $12.5 \mathrm{~mL}$ of iodinated agent). Areas of specific interest were reconstructed using MIP rendering by the senior author in various planes using high-resolution algorithms (voxel sizes, between 0.2 and $0.04 \mathrm{~mm}$ ). ${ }^{3}$

\section{Perimedullary Anastomosis}

Case 1. A superficial anastomosis between the anteromedian spinal vein (AMSV) and the left posterolateral spinal vein (PLSV) was documented at T12 in a 36-year-old woman with progressive myelopathy (Fig 1).

\section{Centrodorsolateral Anastomosis}

Case 2. A centrodorsolateral TMVA connecting the AMSV to the right PLSV at the level of C3 was seen in a 51-year-old woman investigated for a C3-C4 spinal epidural arteriovenous fistula (Fig 2).

\section{Median Anteroposterior Anastomosis}

Case 3. A median anteroposterior TMVA connecting the AMSV to the posteromedian spinal vein (PMSV) at T11 was noted in a 23year-old woman investigated for recurrent myelopathy (Fig 3). 

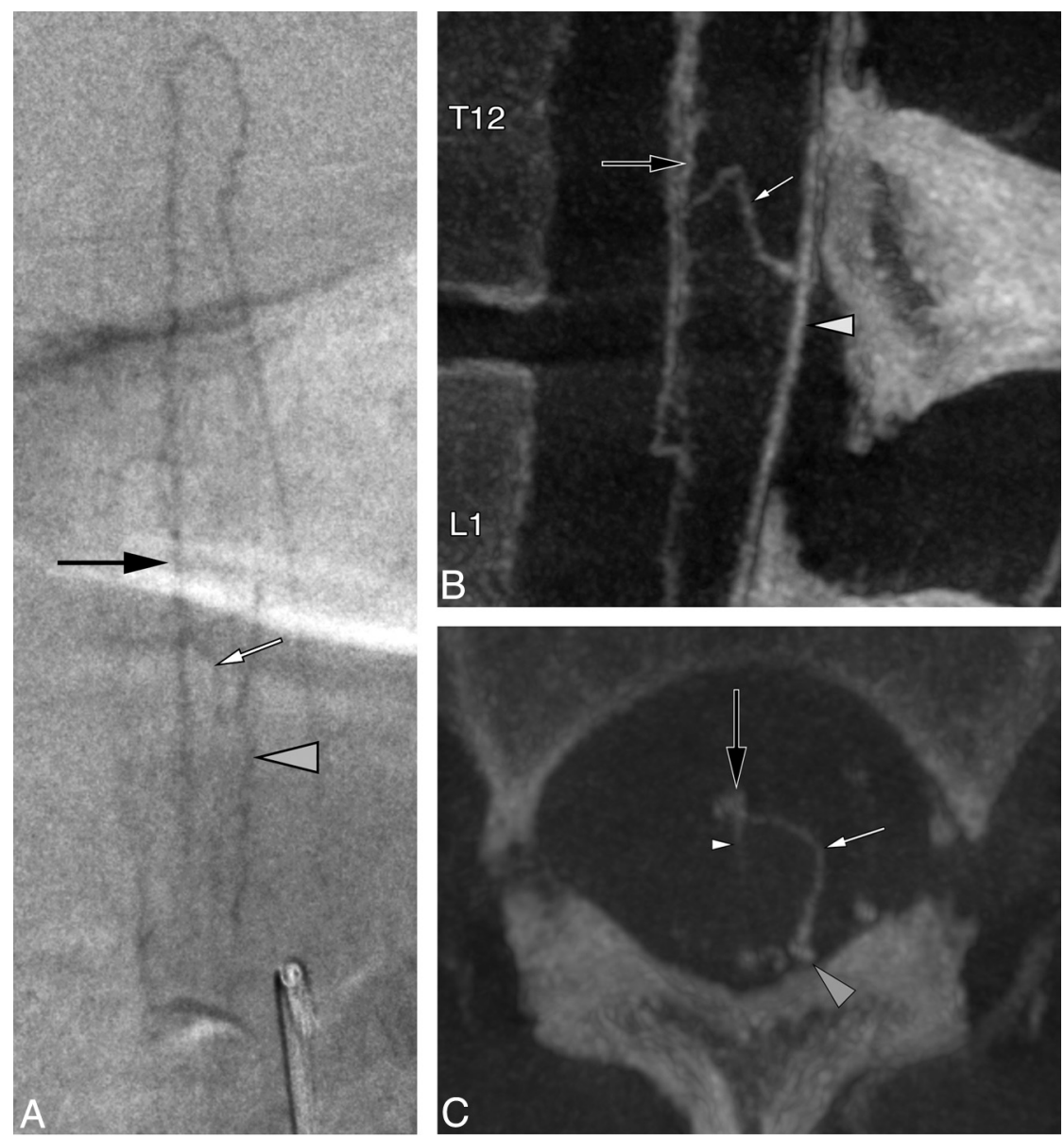

FIG 1. A 36-year-old woman with a perimedullary anastomosis. A, Spinal DSA, left L1 injection, posteroanterior projection, late venous phase, shows an anastomotic connection (white arrow) between the AMSV (black arrow) and the left PLSV (gray arrowhead). The exact location of this anastomosis (intramedullary-versus-perimedullary) cannot be clearly established in this projection. A lateral projection would not offer additional information because the ventrodorsal course of both types of anastomoses would appear similar. B, FPCA, left L1 injection, sagittal MIP reconstruction (thickness $=8 \mathrm{~mm}$ ). The morphology of the anastomosis (white arrow) and its points of connection with the AMSV (black arrow) and left PLSV (gray arrowhead) are better appreciated. C, FPCA, left L1 injection, axial MIP reconstruction (thickness $=8 \mathrm{~mm}$ ). This axial view unequivocally establishes the superficial course of the perimedullary anastomosis (small arrow) over the lateral aspect of the spinal cord, between the AMSV (black arrow) and the left PLSV (gray arrowhead). A central vein is documented within the anteromedian fissure (white arrowhead).
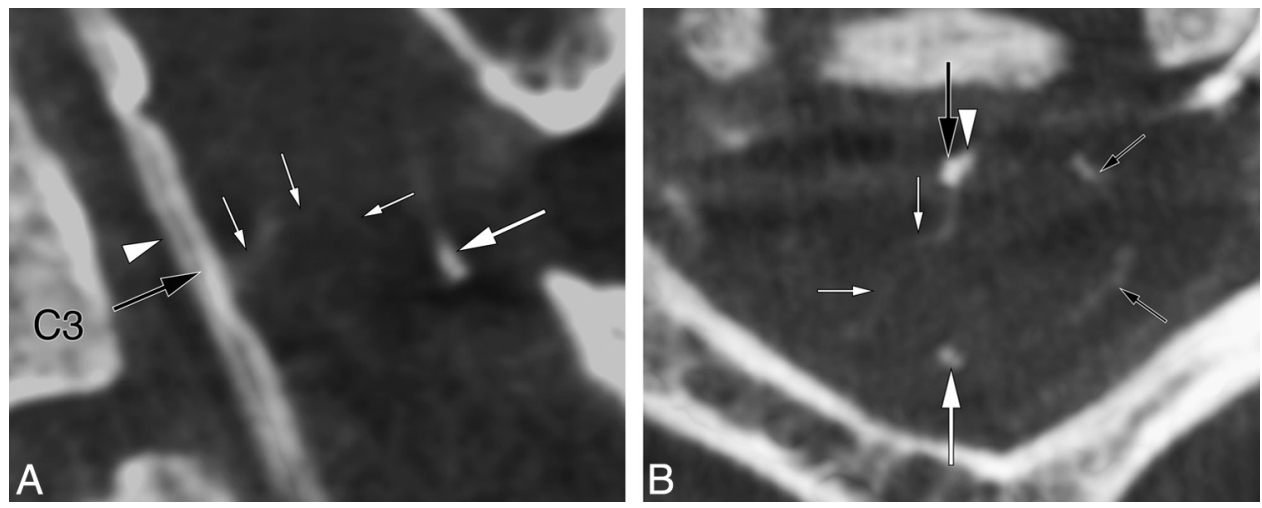

FIG 2. A 51-year-old woman with a centrodorsolateral transmedullary venous anastomosis. A, FPCA, left vertebral artery injection, sagittal MIP reconstruction (thickness $=5 \mathrm{~mm}$ ) shows a centrodorsolateral TMVA (small white arrows) connecting the AMSV (large black arrow) to the right PLSV. The PMSV (large white arrow) and anterior spinal artery (white arrowhead) are also documented. B, FPCA, left vertebral artery injection, axial MIP reconstruction (thickness $=5 \mathrm{~mm}$ ) shows the path taken by the centrodorsolateral TMVA (small white arrows) between the AMSV (large black arrow) and the right PLSV. The PMSV (larger white arrow) and segments of the coronary plexus (small black arrows) are seen as well. 

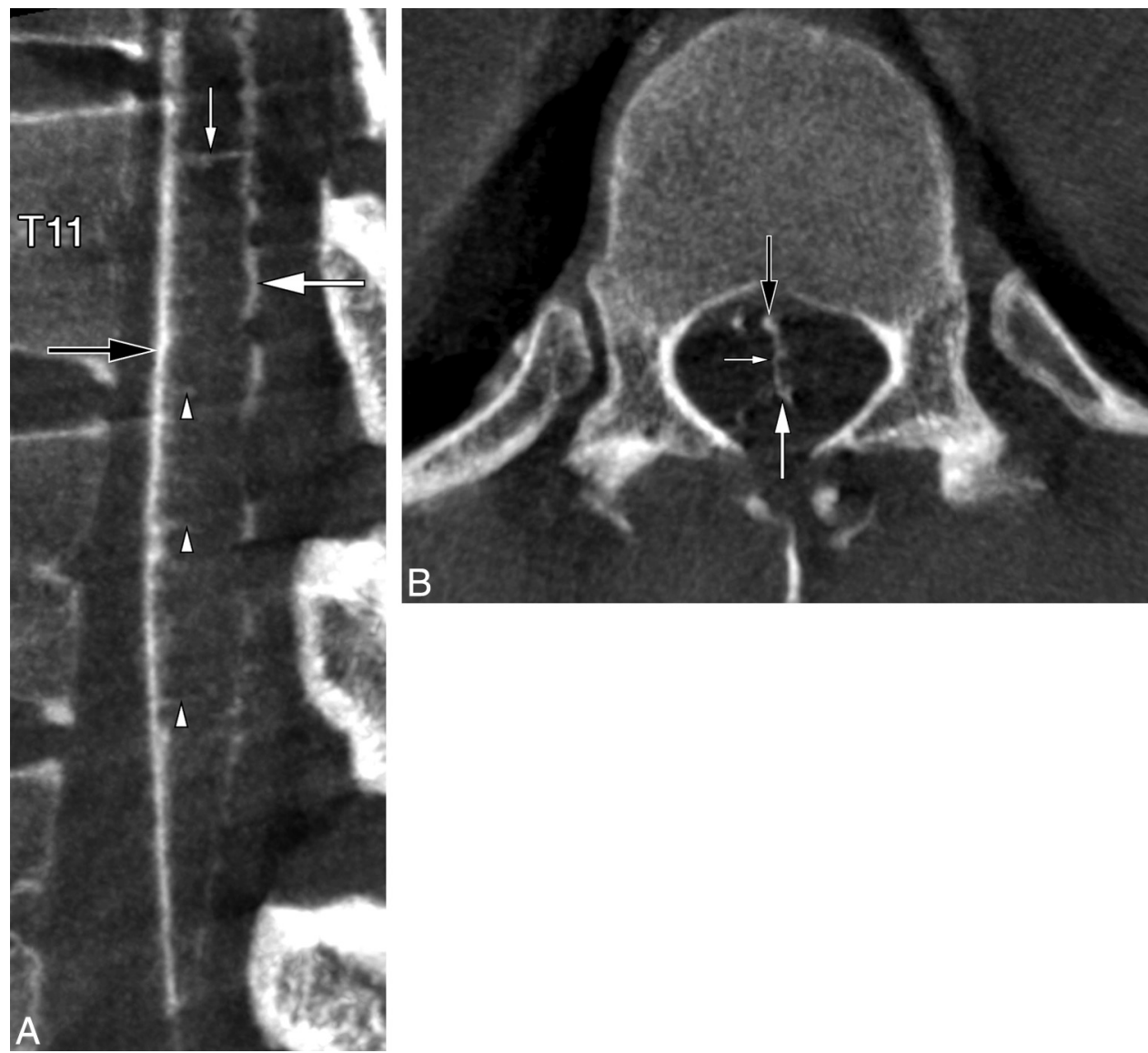

FIG 3. A 23-year-old woman with a median anteroposterior transmedullary venous anastomosis. A, FPCA, right T12 injection, sagittal MIP reconstruction (thickness $=3 \mathrm{~mm}$ ). The median anteroposterior TMVA (small white arrow) extends between the AMSV (black arrow) and the PMSV (large white arrow) at the T11 level. Several central veins (arrowheads) are seen. B, FPCA, right T12 injection, axial MIP reconstruction (thickness $=3 \mathrm{~mm}$ ), confirms the transmedullary course of the median anteroposterior TMVA (small white arrow) between the AMSV (black arrow) and the PMSV (large white arrow).
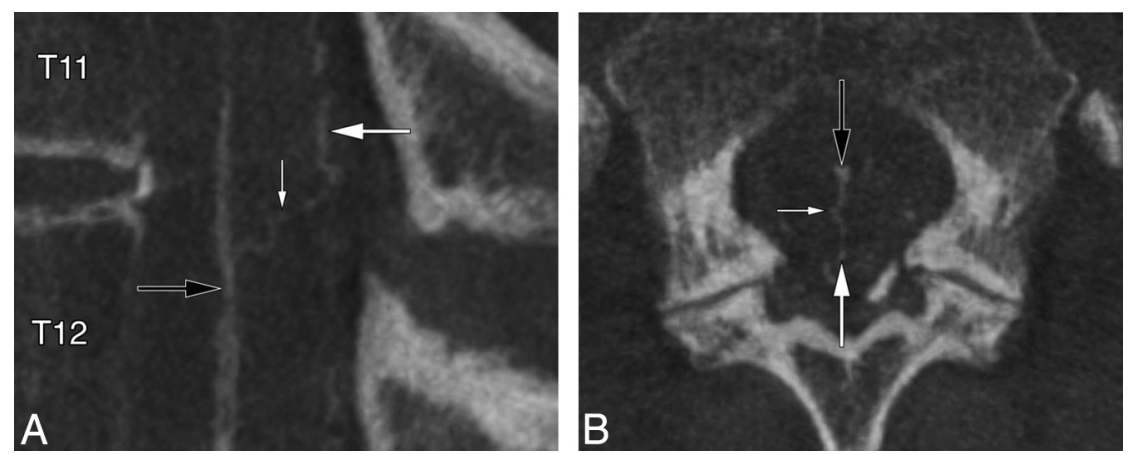

FIG 4. A 60-year-old woman with a median anteroposterior transmedullary venous anastomosis. A, FPCA, left L1 injection, sagittal MIP reconstruction (thickness $=3 \mathrm{~mm}$ ), shows a median anteroposterior TMVA (small white arrow) coursing superiorly and posteriorly between the AMSV (black arrow) and the PMSV (large white arrow). B, FPCA, left L1 injection, axial MIP reconstruction (thickness $=10 \mathrm{~mm}$ ). The median anteroposterior TMVA (small white arrow) extends from the AMSV (black arrow) to the PMSV (large white arrow). Note the typical lateral loop of the TMVA around the central canal at the level of the small white arrow.

Case 4. A median anteroposterior TMVA was documented at the T11-12 level in a 60-year-old woman investigated for progressive myelopathy (Fig 4).

Case 5. A median anteroposterior TMVA was seen at T11 in a 74-year-old man investigated for progressive myelopathy (Fig 5).

Case 6. A median anteroposterior TMVA was noted at C7 in a 40year-old woman with progressive myelopathy (Fig 6).

\section{Combined Anastomoses}

Case 7. A 52-year-old man with progressive myelopathy was treated endovascularly for 2 spinal epidural AVFs (left T7 and T9). FPCA performed to investigate an additional cervical spinal epidural AVF showed a median anteroposterior TMVA with a duplicate origin from the AMSV at the C2 level, a combined centrodorsolateral and median anteroposterior TMVA between C2 and C3, and a 

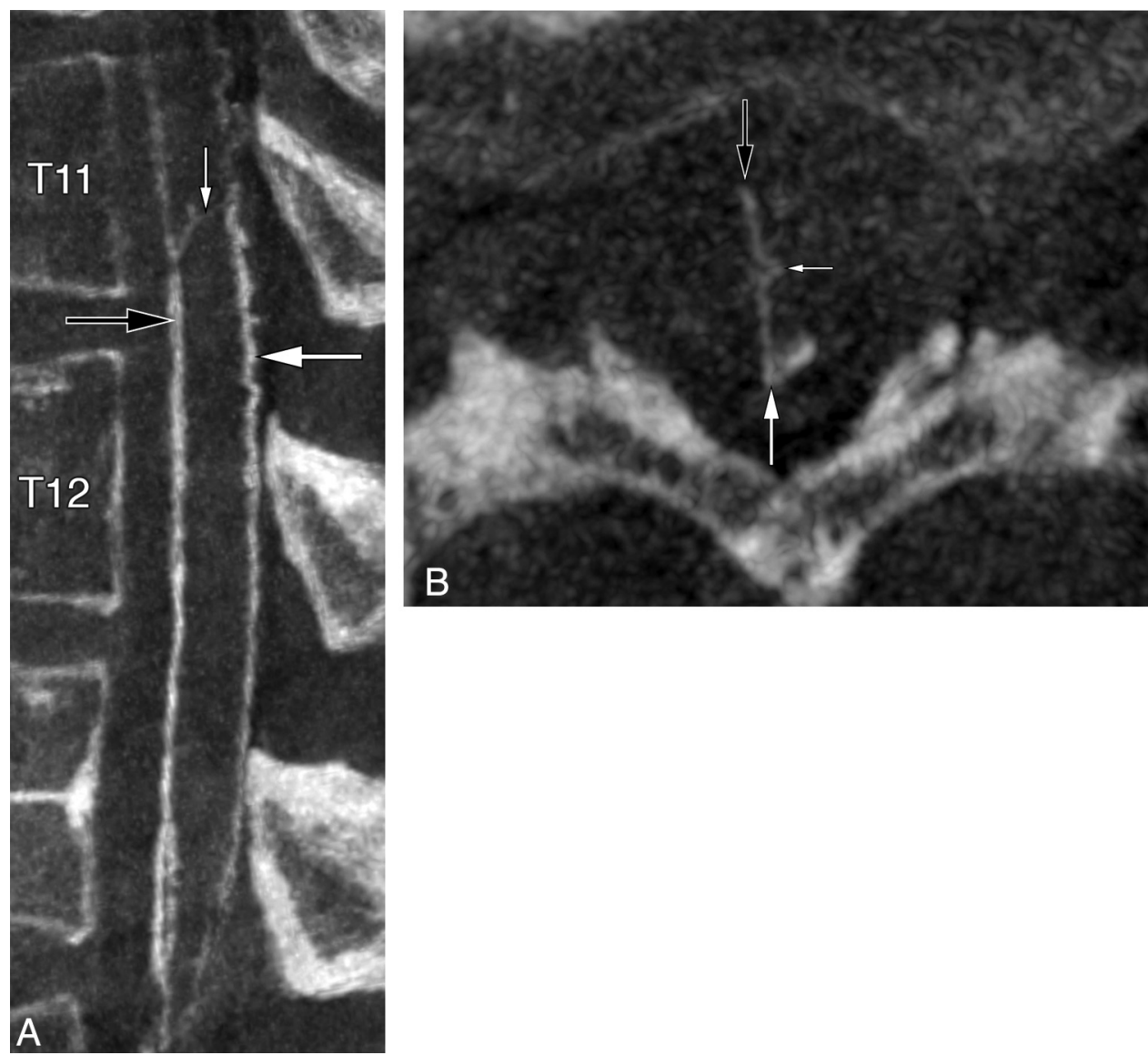

FIG 5. A 74-year-old man with a median anteroposterior transmedullary venous anastomosis. A, FPCA, left L1 injection, sagittal MIP reconstruction (thickness $=3 \mathrm{~mm}$ ), shows a median anteroposterior TMVA (small white arrow) extending between the AMSV (black arrow) and the PMSV (large white arrow). B, FPCA, left L1 injection, axial MIP reconstruction (thickness $=10 \mathrm{~mm}$ ). The median anteroposterior TMVA (small white arrow) courses from the AMSV (black arrow) to the PMSV (large white arrow), showing a typical deviation around the central canal.
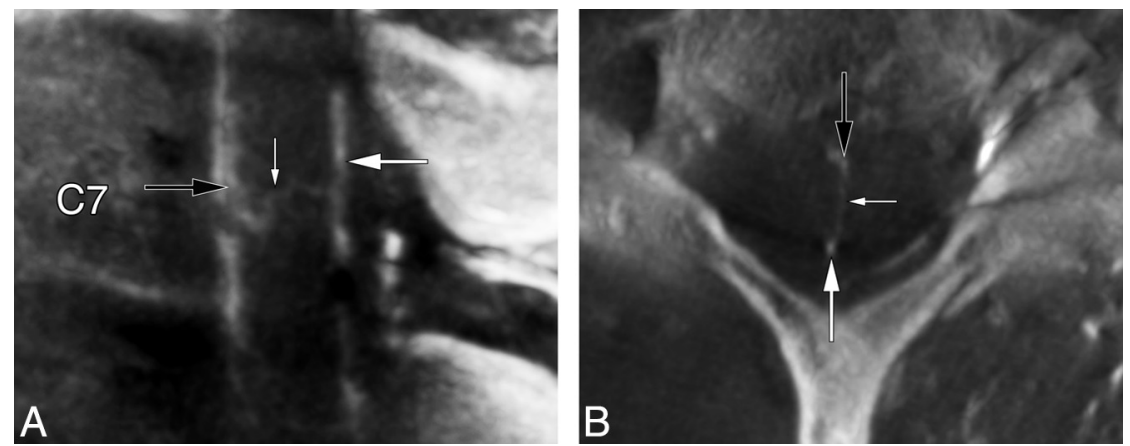

FIG 6. A 40-year-old woman with a median anteroposterior transmedullary venous anastomosis. A, FPCA, left costocervical trunk injection, sagittal MIP reconstruction (thickness $=2 \mathrm{~mm}$ ). A median anteroposterior TMVA (small white arrow) connects the AMSV (black arrow) to the PMSV (large white arrow). B, FPCA, left costocervical trunk injection, axial MIP reconstruction (thickness $=4 \mathrm{~mm}$ ), confirms the intramedullary trajectory of the median anteroposterior TMVA (small white arrow) between the AMSV (black arrow) and the PMSV (large white arrow).

median anteroposterior TMVA between C3 and C4, associated with a sulcal longitudinal anastomosis (Fig 7).

\section{Pathologic Illustration}

Case 8. A 57-year-old woman was investigated for a cervical stroke possibly secondary to a C3-C4 disk herniation. FPCA performed before surgery confirmed the presence of mass effect on the anterior spinal vessels by disk material, matching the location of the ischemic lesion on MR imaging. The central veins were barely visible at the compression site yet were prominent immediately above and below it. A sulcal longitudinal anastomosis served as a collateral venous pathway in the depth of the anteromedian fissure, and several TMVAs were noted in neighboring areas, including 2 centrodorsolateral TMVAs respectively located cranially and caudally to the compression site (Fig 8). 

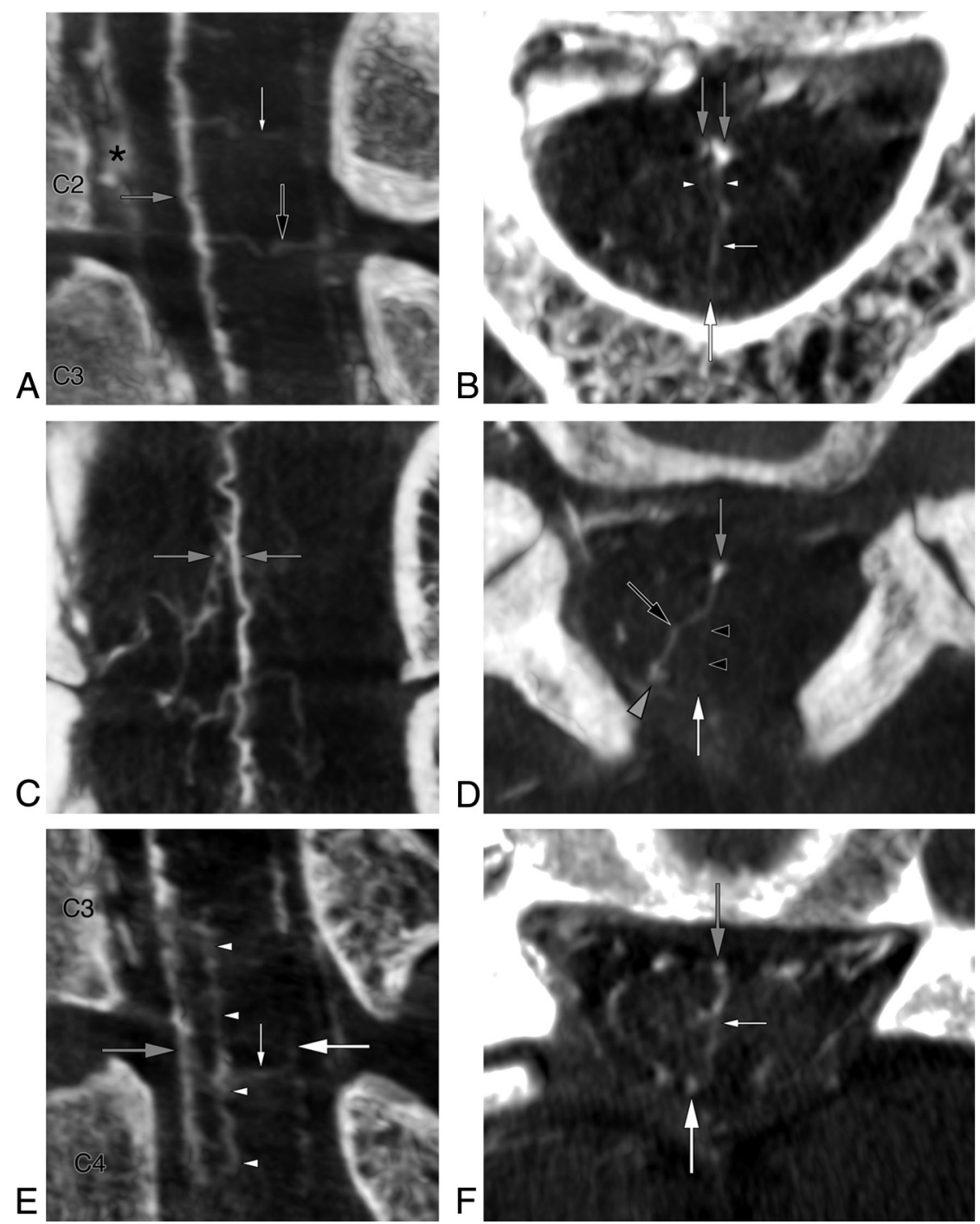

FIG 7. A 52-year-old man with 2 median anteroposterior transmedullary venous anastomoses and a combined centrodorsolateral and median anteroposterior TMVA. A, FPCA, right vertebral injection, sagittal MIP reconstruction (thickness $=5 \mathrm{~mm}$ ), reveals 2 TMVAs: a median anteroposterior TMVA (small white arrow) with a dual origin (documented in Fig 7B) from the AMSV (gray arrow) at C2 and a TMVA with combined median anteroposterior and centrodorsolateral characteristics (black arrow) between C2 and C3. The anterior internal epidural venous plexus is well-appreciated (asterisk). B, FPCA, right vertebral injection, axial MIP reconstruction (thickness $=3 \mathrm{~mm}$ ), shows the median anteroposterior TMVA (small white arrow) at the C2 level, with a dual origin (arrowheads) from the AMSV and the surrounding venous network (gray arrows). The PMSV (large white arrow) is seen as well. C, FPCA, right vertebral injection, coronal MIP reconstruction (thickness $=4 \mathrm{~mm}$ ), documents the segment of the AMSV and surrounding venous network from which the TMVA with dual roots originates (gray arrows). D, FPCA, right vertebral injection, axial MIP reconstruction (thickness $=4 \mathrm{~mm}$ ). The TMVA with combined median anteroposterior (black arrowheads) and centrodorsolateral (black arrow) characteristics connects the AMSV (gray arrow) to the PMSV dorsally (white arrow) and to the right PLSV dorsolaterally (gray arrowhead) between the C2 and C3 levels. E, FPCA, right vertebral injection, sagittal MIP reconstruction (thickness $=4 \mathrm{~mm}$ ). A median anteroposterior TMVA (small white arrow) extends between the AMSV (gray arrow) and the PMSV (large white arrow). A sulcal longitudinal anastomosis (arrowheads) is seen within the depth of the anteromedian fissure between C3 and C4. F, FPCA, right vertebral injection, axial MIP reconstruction (thickness $=3 \mathrm{~mm}$ ), shows the course of the median anteroposterior TMVA (small white arrow) between the AMSV (gray arrow) and the PMSV (large white arrow).

\section{DISCUSSION \\ FPCA Acquisitions}

All FPCA acquisitions were successful, with no intra- or postprocedural complications. FPCA is primarily performed in our practice to complement angiograms with negative findings in patients with progressive myelopathies and MR imaging findings suggesting venous hypertension, which explains the high ratio of studies with normal findings ( 5 of 8 ). 3D spinal DSA is the preferred technique for the evaluation of fast-flow lesions such as arteriovenous malformations or perimedullary arteriovenous fistulas, while FPCA is occasionally performed for slow-flow lesions (eg, spinal epidural AVFs). Finally, the spinal venous system is not specifically studied when FPCA is performed to investigate an arterial condition (crus compression syndrome, for example).

The principal advantage of FPCA over other vascular imaging techniques such as CTA or MRA is its higher spatial resolution. ${ }^{3}$ As a drawback of its acquisition mode, FPCA will display arteries and veins simultaneously. These can, however, be differentiated on the basis of their course, branching pattern, and correlation with the corresponding $2 \mathrm{D}$ spinal DSA anatomy. ${ }^{3}$

AJNR Am J Neuroradiol 36:1381-88 Jul 2015 www.ajnr.org 


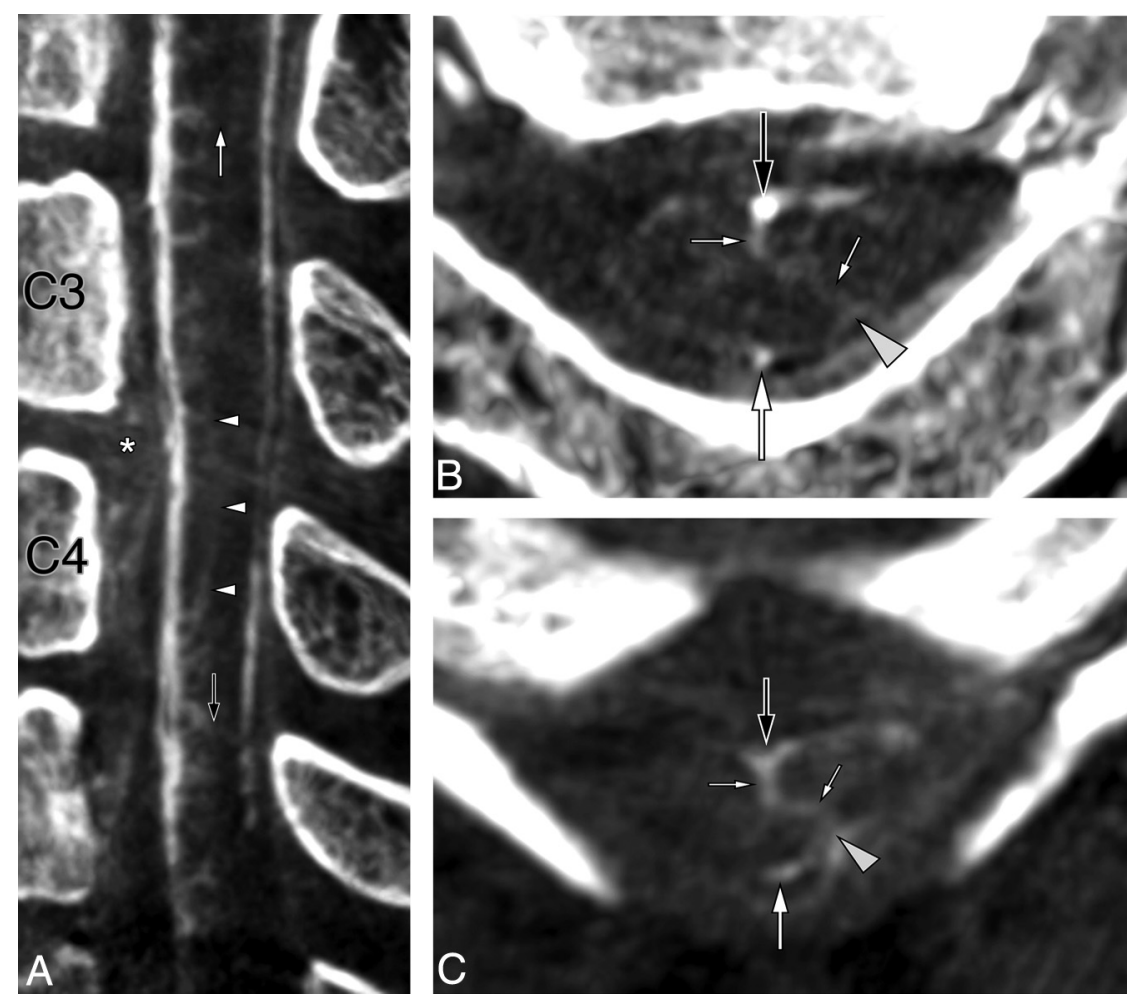

FIG 8. A 57-year-old woman with a sulcal longitudinal anastomosis and 2 centrodorsolateral transmedullary venous anastomoses. A, FPCA, left vertebral injection, sagittal MIP reconstruction (thickness $=5 \mathrm{~mm}$ ), shows mass effect on the anterior aspect of the spinal cord by protruded disk material at C3-C4 (asterisk), with a dearth of central veins at the corresponding level. The central veins above and below the lesion are prominent. A sulcal longitudinal anastomosis (white arrowheads) appears to serve as a collateral venous pathway in the depth of the anteromedian fissure. Two centrodorsolateral TMVAs are noted, respectively located cranially (white arrow) and caudally (black arrow) to the compressed area. B, FPCA, left vertebral injection, axial MIP reconstruction (thickness $=3 \mathrm{~mm}$ ), shows the C2-C3 centrodorsolateral TMVA (small white arrows) connecting the AMSV (large black arrow) to the left PLSV (gray arrowhead). The PMSV (large white arrow) is seen as well. $C$, FPCA, left vertebral injection, axial MIP reconstruction (thickness $=2 \mathrm{~mm}$ ), documents a second centrodorsolateral TMVA (small white arrows) at C4-C5, extending between the AMSV (large black arrow) and left PLSV (gray arrowhead), which continues its course medially to connect with the PMSV (large white arrow).

\section{Anatomy of the Perimedullary and Intramedullary Venous Systems}

The perimedullary venous system includes the AMSV and PMSV, which are 2 anastomotic chains respectively coursing over the anteromedian and posteromedian fissures. The smaller paired PLSVs lie along the dorsal nerve rootlets (Fig 9A). ${ }^{9,10}$ The principal longitudinal venous axes are interconnected by the coronary plexus of the pia mater. ${ }^{11}$ The coronary plexus generally includes a few larger perimedullary anastomoses that can be visualized during spinal DSA, if the venous phase is of sufficient quality. However, perimedullary anastomoses are not clearly distinguished from TMVAs without the multiplanar assistance of FPCA (Fig 1$).^{3}$

The intramedullary venous network is divided into central and peripheral compartments (Fig 9A). The central veins drain the anterior and median portions of the spinal parenchyma ${ }^{12}$ and terminate into the AMSV. Neighboring central veins can establish longitudinal anastomotic chains, both within the central gray matter (paracentral longitudinal anastomosis) and the anteromedian fissure (sulcal longitudinal anastomosis). ${ }^{12}$ Paracentral longitudinal anastomoses can participate in aberrant intramedullary drainage patterns similar to the developmental venous anomalies seen in the brain or brainstem. ${ }^{4}$ When prominent, sulcal or paracentral longitudinal anastomoses can be visualized with FPCA (Fig 7E).
The peripheral veins course radially to end in the coronary plexus. Those located within the anterior and posterior radicular fasciculi are generally more developed. ${ }^{13}$ The septal veins drain into the PMSV. All intrinsic veins drain centrifugally into the perimedullary venous system. ${ }^{14,15}$

\section{Transmedullary Venous Anastomoses}

Two types of TMVAs are classically recognized. The centrodorsolateral type was described by Kadyi ${ }^{11}$ in 1889 (Fig 10):

A large anastomotic connection is established between one of the central veins and one from the posterior surface of the spinal cord. The small trunk designated by " $v$ " does not course within the median septum, but leftward to reach the surface of the spinal cord in the vicinity of the line of emergence of the left posterior nerve rootlets... The venous anastomosis " $a$ " forms a loop, both legs of which run parallel to the central canal: one goes down, the other one goes up and follows a S-shaped curve before reaching the trunk of the corresponding central vein " $c$ " (p 146, P.G. translation).

In 1939, Herren and Alexander ${ }^{14}$ further defined the centrodorsolateral anastomosis as being formed by a central vein connecting to a paracentral vein, itself continuing as a long oblique venous channel that terminates in the ipsilateral PLSV (Fig 9B). 

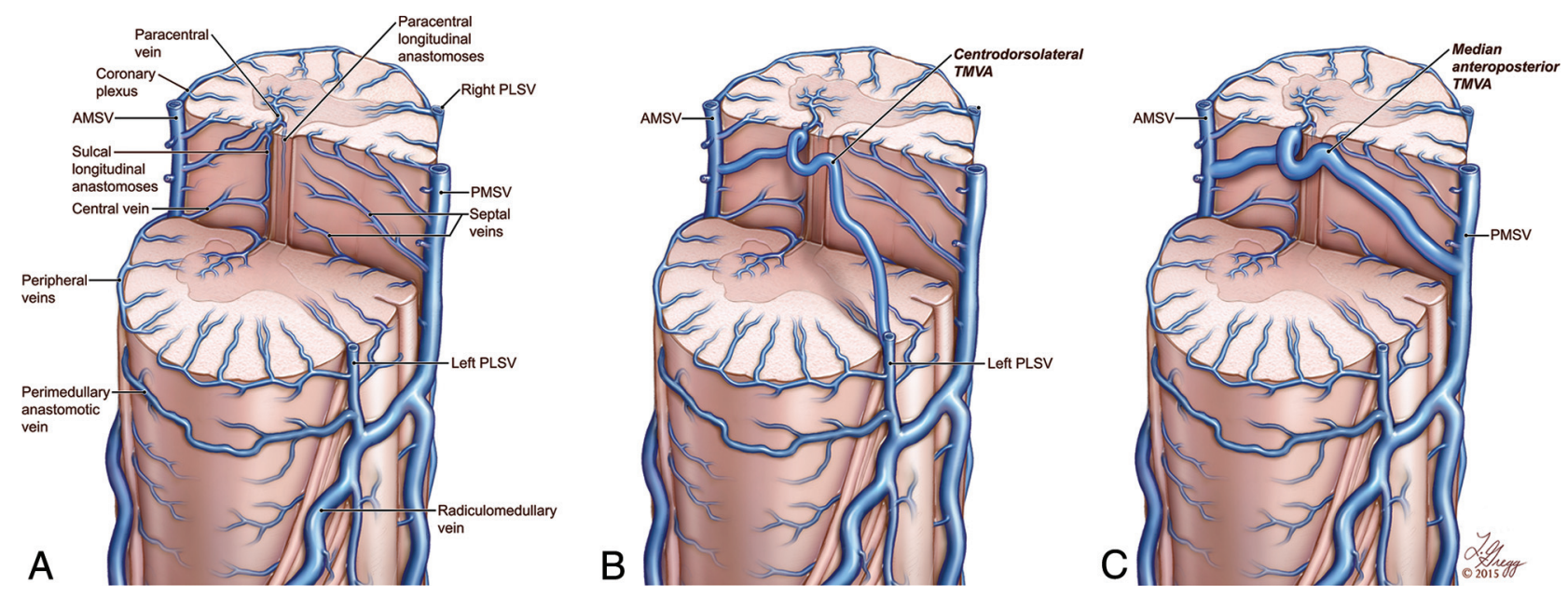

FIG 9. Artistic representation of the normal anatomy of the spinal venous system, emphasizing the 2 classic types of transmedullary venous anastomoses. $A$, The central veins establish longitudinal connections within the central gray matter (paracentral longitudinal anastomosis) and in the depth of the anteromedian fissure (sulcal longitudinal anastomosis). B, A centrodorsolateral TMVA is formed by a central vein connecting to a paracentral vein and continuing as a long oblique venous channel joining the ipsilateral PLSV. C, A median anteroposterior TMVA is formed by a large connection established between a central vein and a septal vein, with a typical loop around the central canal. Copyright 2015 Lydia Gregg.

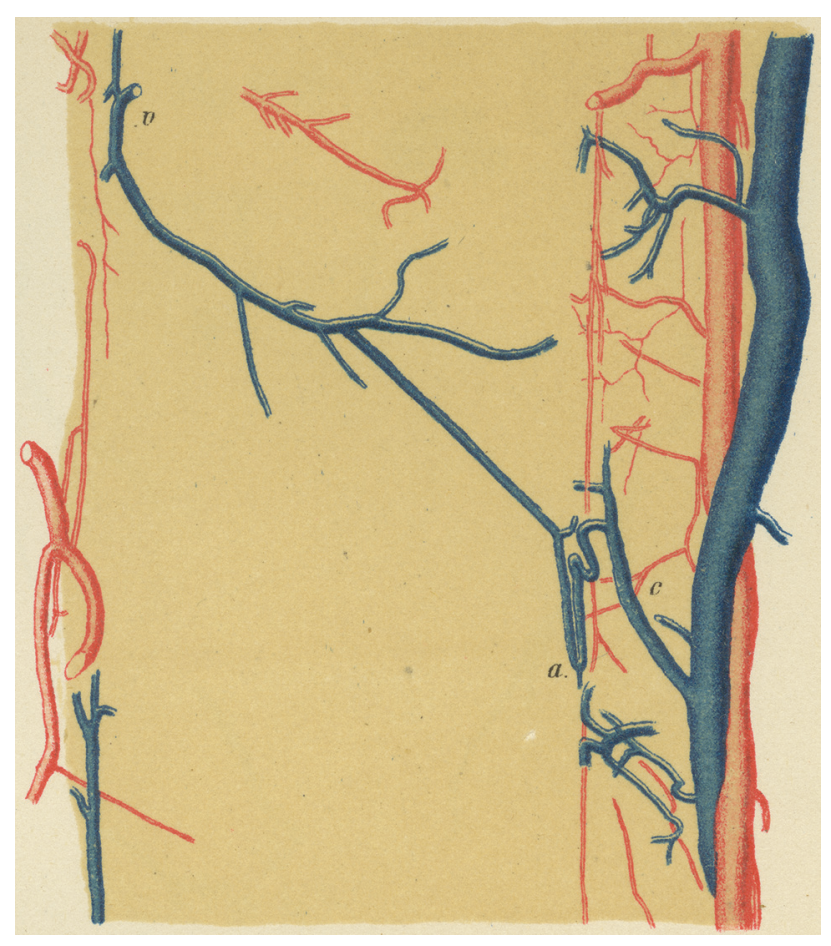

FIG 10. Illustration reproduced from Kadyi H. Über die Blutgefässe des Menschlichen Rückenmarkes. Lemberg: Gubryonowisz \& Schmidt; 1889 (Fig 9, page 146) shows the first TMVA depiction known to the authors (ie, a sagittal view of a centrodorsolateral TMVA). The vein, designated as $v$, courses laterally and posteriorly from the midsagittal plane to a PLSV. The segment $a$ connects to a central vein $c$ after looping around the central canal.

They specifically named this last oblique portion "centrodorsolateral anastomosis," while Crock and Yoshizawa ${ }^{16}$ applied the term to the whole anastomotic chain, from the AMSV to the PLSV.

Crock and Yoshizawa ${ }^{16}$ described a second type of TMVA linking the AMSV to the PMSV, the median anteroposterior anastomosis (Fig 9C). This type of TMVA probably also derives from a connection established between a central vein and a paracentral vein, which then continues dorsally as a septal vein rather than adopting a dorsolateral course. ${ }^{16}$

Case 7 revealed 2 previously unreported TMVA configurations. The first was a median anteroposterior anastomosis with a duplicated origin from the AMSV and from the surrounding venous network (Fig 7B); the second combined median anteroposterior and centrodorsolateral characteristics, draining both dorsally into the PMSV and dorsolaterally into a PLSV (Fig 7D).

While initially believed to be rare, ${ }^{12}$ both types of TMVAs were consistently documented in a microangiographic study of postmortem material, though with irregular sizes and topographic distributions. ${ }^{15}$ The highest prevalence was noted in the upper cervical and cervicothoracic regions (up to 2 TMVAs per centimeter). The largest channels were also found in the cervicothoracic region. ${ }^{15}$ The distance separating 2 TMVAs was increased in the lower thoracic region, while no TMVAs were noted in the lumbar region. They were occasionally documented at the level of the conus medullaris, either supplementing or replacing the anastomotic venous circle of the conus. ${ }^{15}$ Centrodorsolateral TMVAs were more frequent but generally smaller $(0.1-0.2 \mathrm{~mm})$ than median anteroposterior TMVAs $(0.3-0.7 \mathrm{~mm}) .{ }^{15}$ Both types can be sinuous or can adopt a relatively straight intramedullary path. ${ }^{15}$ The relation between the size, number, and course of TMVAs and factors such as age and sex is currently unknown.

TMVAs receive relatively few intramedullary tributaries even though they are generally comparable in size with the principal longitudinal venous axes. ${ }^{14,15}$ Herren and Alexander ${ }^{14}$ proposed that rather than having a drainage function, TMVAs might help regulate pressure and flow by rapidly transferring blood from one longitudinal axis to another. ${ }^{14}$ This hypothesis was later supported by Thron and Rossberg, ${ }^{15}$ who suggested a correlation between the higher cervical and cervicothoracic prevalence of TMVAs and the greater motion range of the spine and spinal cord at these levels. TMVAs might thus protect the principal venous axes from pressure variations related to compression occurring during hyperflexion and hyperextension of the cervical spine. ${ }^{15}$ 
Our last example (case 8) suggests that under abnormal circumstances leading to impaired venous flow, TMVAs may indeed act as alternate drainage pathways limiting the risk of venous engorgement and ischemia (Fig 8). In this patient, a sulcal longitudinal anastomosis also appeared to play the role of a collateral pathway in the area compressed by protruding disk material.

Recognizing TMVAs as normal structures carries both diagnostic and therapeutic implications. For example, TMVAs were recently used as an access route for successful obliteration of a perimedullary arteriovenous fistula. ${ }^{8}$ Median anteroposterior TMVAs have been documented by contrast-enhanced MRA and by DSA, ${ }^{6,7,17}$ while centrodorsolateral TMVAs have, to our knowledge, not been imaged clinically until now, probably because of their smaller size. ${ }^{15}$ New imaging methods such as spinal SWI also require a sound understanding of the spinal venous system anatomy and its variants, for example, to differentiate TMVAs from small intraparenchymal hemorrhages. ${ }^{18}$

In summary, the introduction of novel imaging techniques, such as spinal FPCA or SWI, can strengthen our still deficient understanding of medullary venous pathology. This improved imaging ability creates the need for a more intricate knowledge of the anatomy of the spinal venous system. This article reports several types of TMVAs documented by FPCA. Some of these vessels were previously known, in general from postmortem investigations, while others had not yet been described. Appreciating the existence of TMVAs is clinically important because these channels may be confused with intramedullary hemorrhages or vascular malformations on noninvasive imaging.

Disclosures: Philippe Gailloud_UNRELATED: Consultancy: Codman Neurovascular; Grants/Grants Pending: Siemens Medical*; Stock/Stock Options: Artventive Medical; Other: Consultant for Penumbra. *Money paid to the institution.

\section{REFERENCES}

1. Akpek S, Brunner T, Benndorf G, et al. Three-dimensional imaging and cone beam volume $\mathrm{CT}$ in $\mathrm{C}$-arm angiography with flat panel detector. Diagn Interv Radiol 2005;11:10-13

2. Zellerhoff M, Scholz B, Ruehrnschopf EP, et al. Low contrast 3D reconstruction from C-arm data. SPIE Proceedings 2005;5745: $646-55$

3. Chen J, Ethiati T, Gailloud P. Flat panel catheter angiotomography of the spinal venous system: an enhanced venous phase for spinal digital subtraction angiography. AJNR Am J Neuroradiol 2012; 33:1875-81

4. Pearl MS, Chen JX, Gregg L, et al. Angiographic detection and characterization of "cryptic venous anomalies" associated with spinal cord cavernous malformations using flat-panel catheter angiotomography. Neurosurgery 2012;71:125-32

5. Aadland TD, Thielen KR, Kaufmann TJ, et al. 3D C-arm conebeam $\mathrm{CT}$ angiography as an adjunct in the precise anatomic characterization of spinal dural arteriovenous fistulas. AJNR Am J Neuroradiol 2010;31:476-80

6. Thron AK. Vascular anatomy of the spine and spinal cord. In: Hurst RW, Rosenwasser RH, eds. Neurointerventional Management: Diagnosis and Treatment. Boca Raton, FL: CRC Press; 2012:40-58

7. Krings T. Vascular malformations of the spine and spinal cord*: anatomy, classification, treatment. Clin Neuroradiol 2010;20:5-24

8. Giese A, Winkler PA, Schichor C, et al. A transmedullary approach to occlusion of a ventral perimedullary arteriovenous fistula of the thoracic spinal cord. Neurosurgery 2010;66:611-15

9. Suh T, Alexander L. Vascular system of the human spinal cord. Arch Neurol Psychiatry 1939;41:659-77

10. Zhang ZA, Nonaka H, Hatori T. The microvasculature of the spinal cord in the human adult. Neuropathology 1997;17:32-42

11. Kadyi H. Über die Blutgefüsse des Menschlichen Rückenmarkes. Lemberg: Gubryonowisz \& Schmidt; 1889

12. Gillilan LA. Veins of the spinal cord: anatomic details—suggested clinical applications. Neurology 1970;20:860-68

13. Lazorthes G, Gouaze A, Djindjian R. Vascularisation et circulation de la moelle épinière: anatomie, physiologie, pathologie, angiographie. Paris: Masson \& Cie; 1973

14. Herren RY, Alexander L. Sulcal and intrinsic blood vessels of the human spinal cord. Arch Neurol Psychiatry 1939;41:678-87

15. Thron AK, Rossberg C, Mironov A. Vascular Anatomy of the Spinal Cord: Neuroradiological Investigations and Clinical Syndromes. New York: Springer-Verlag Wien; 1988

16. Crock HV, Yoshizawa H. The Blood Supply of the Vertebral Column and Spinal Cord in Man. New York: Springer-Verlag; 1977

17. Thron A, Mull M. Blood vessels of the spinal cord: anatomical and MR-imaging correlation. Rivista di Neuroradiologia 2004;17: 277-81

18. Ishizaka K, Kudo K, Fujima N, et al. Detection of normal spinal veins by using susceptibility-weighted imaging. J Magn Reson Imaging 2010;31:32-38 